IJMMS 27:6 (2001) 387-389

PII. S0161171201010997

http://ijmms.hindawi.com

(c) Hindawi Publishing Corp.

\title{
FINITE AG-GROUPOID WITH LEFT IDENTITY AND LEFT ZERO
}

\author{
QAISER MUSHTAQ and M. S. KAMRAN
}

(Received 3 October 2000)

\begin{abstract}
A groupoid $G$ whose elements satisfy the left invertive law: $(a b) c=(c b) a$ is known as Abel-Grassman's groupoid (AG-groupoid). It is a nonassociative algebraic structure midway between a groupoid and a commutative semigroup. In this note, we show that if $G$ is a finite AG-groupoid with a left zero then, under certain conditions, $G$ without the left zero element is a commutative group.
\end{abstract}

2000 Mathematics Subject Classification. 20N99.

1. Preliminaries. An Abel-Grassman's groupoid [6], abbreviated as AG-groupoid, is a groupoid $G$ whose elements satisfy the left invertive law: $(a b) c=(c b) a$. It is also called a left almost semigroup [2, 3, 4, 5]. In [1], the same structure is called left invertive groupoid. In this note we call it AG-groupoid.

It is a nonassociative algebraic structure midway between a groupoid and a commutative semigroup. The structure is medial [5], that is, $(a b)(c d)=(a c)(b d)$ for all $a, b, c, d \in G$. It has been shown in [5] that if an AG-groupoid contains a left identity then it is unique. It has been proved also that an AG-groupoid with right identity is a commutative monoid, that is, a semigroup with identity element. An element $a_{0}$ of an AG-groupoid $G$ is called a left (right) zero if $a_{0} a=a_{0}\left(a a_{0}=a_{0}\right)$ for all $a \in G$.

Let $a, b, c$, and $d$ belong to an AG-groupoid with left identity and $a b=c d$. Then it has been shown in [5] that $b a=d c$.

An element $a^{-1}$ of an AG-groupoid with left identity $e$ is called a left inverse if $a^{-1} a=e$. It has been shown in [5] that if $a^{-1}$ is a left inverse of $a$ then it is unique and is also the right inverse of $a$.

If for all $a, b, c$ in an AG-groupoid $G, a b=a c$ implies that $b=c$, then $G$ is known as left cancellative. Similarly, if $b a=c a$, implies that $b=c$, then $G$ is called right cancellative. It is known [5] that every left cancellative AG-groupoid is right cancellative but the converse is not true. However, every right cancellative AG-groupoid with left identity is left cancellative.

In this note, we show that if $G$ is a finite AG-groupoid with left identity and a left zero $a_{0}$, under certain conditions $G \backslash\left\{a_{0}\right\}$ is a commutative group without a left zero.

2. Results. We need the following theorem from [4] for our main result.

THEOREM 2.1 [4]. A cancellative AG-groupoid $G$ is a commutative semigroup if $a(b c)=(c b) a$ for all $a, b, c \in G$. 
We now state and prove our main result.

THEOREM 2.2. Let $(G, \circ)$ be a finite AG-groupoid with at least two elements. Suppose that it contains a left identity and a left zero $a_{0}$. Then $G^{0}=G \backslash\left\{a_{0}\right\}$ is a commutative group under the binary operation ( $\circ)$ provided there is another binary operation $(*)$ such that

(i) $(G, *)$ is an $A G$-groupoid with left identity and left inverses,

(ii) $a_{0} * a=a$, for all $a \in G$,

(iii) $(a * b) \circ c=(a \circ c) *(b \circ c)$, for all $a, b, c \in G$,

(iv) $a \circ b=a_{0}$ implies that either $a=a_{0}$ or $b=a_{0}$ for all $a, b \in G$,

(v) $a \circ(b \circ c)=(c \circ b) \circ a$, for all $a, b, c \in G$.

Proof. Suppose that $G=\left\{a_{0}, a_{1}, \ldots, a_{m}\right\}$, where $m$ is a positive integer, is an AGgroupoid with left identity under the binary operation $(\circ)$. Let $e$ be the identity element of $G$. It is certainly different from $a_{0}$ because of (ii) and because $a_{0}$ is the left zero under ( $\circ)$. The left invertive law together with (iv) implies that $\left(a \circ a_{0}\right) \circ e=\left(e \circ a_{0}\right) \circ a=$ $a_{0} \circ a=a_{0}$, where $e \neq a_{0}$. That is,

$$
a_{0} \circ a=a \circ a_{0}=a_{0} .
$$

Now consider the subset $G^{0}$ of $G$ which is obtained from it by deleting $a_{0}$, so that $G^{0}=\left\{a_{i}: i=1,2, \ldots, m\right\}$. In view of the facts that $a_{0}$ is a zero under the binary operation $(\circ)$ and it is the left identity under $(*)$ and that $(G, \circ)$ is a finite AG-groupoid with left identity. $\left(G^{0}, \circ\right)$ is also a finite AG-groupoid with left identity having the same $e$ as the left identity in which all elements are distinct.

We now examine whether an element $a$ of $G^{0}$ has an inverse in $G^{0}$ under (o) or not. We construct a set $H_{k}=\left\{a_{k} \circ a_{1}, a_{k} \circ a_{2}, \ldots, a_{k} \circ a_{m}\right\}$, where $a_{k} \neq a_{0}$. If $a_{k}=a_{0}$, then because $a_{0}$ is a left zero in $G$ under ( $\left.\circ\right)$ and the left identity under $(*)$, the ultimate form of the set $H_{k}$ will be $\left\{a_{0}\right\}$. Therefore it validates our supposition that $a_{k} \neq a_{0}$.

We assert that $H_{k}$ contains $m$ elements. Suppose otherwise and let

$$
a_{k} \circ a_{r}=a_{k} \circ a_{s},
$$

for some $r, s=1,2, \ldots, m$ and $r \neq s$. Since $H_{k}$ is an AG-groupoid with left identity under (०), therefore (2.2) implies that

$$
a_{r} \circ a_{k}=a_{s} \circ a_{k},
$$

for some $r, s=1,2, \ldots, m$ and $r \neq s$. Consider now the element $\left(a_{s} * a_{r}^{-1}\right) \circ a_{k}$, which is certainly an element of $G$, where $a_{r}^{-1}$ is the left inverse of $a_{r}$ in $G$ with respect to $(*)$. Now,

$$
\begin{aligned}
\left(a_{s} * a_{r}^{-1}\right) \circ a_{k} & =\left(a_{s} \circ a_{k}\right) *\left(a_{r}^{-1} \circ a_{k}\right)=\left(a_{r} \circ a_{k}\right) *\left(a_{r}^{-1} \circ a_{k}\right) \\
& =\left(a_{r} * a_{r}^{-1}\right) \circ a_{k}=a_{0} \circ a_{k}=a_{0} .
\end{aligned}
$$

Because of (iii), equation (2.3) and the facts that $a_{r}^{-1}$ is the inverse of $a_{r}$ under (*). Thus $\left(a_{s} * a_{r}^{-1}\right) \circ a_{k}=a_{0}$. Since $a_{k} \neq a_{0}$, therefore because of (iv), $a_{s} * a_{r}^{-1}=a_{0}$. Next $\left(a_{s} * a_{r}^{-1}\right) \circ a_{r}=a_{0} * a_{r}$ implies that $\left(a_{s} * a_{r}^{-1}\right) \circ a_{r}=a_{r}$ because $a_{0}$ is the left identity in $G$ under $(*)$. Hence, $a_{r}=\left(a_{s} * a_{r}^{-1}\right) * a_{r}=\left(a_{r} * a_{r}^{-1}\right) * a_{s}=a_{0} * a_{s}=a_{s}$, that is, $a_{r}=a_{s}$. Since $\left|H_{k}\right|=m$, therefore the result $a_{r}=a_{s}$ contradicts our assumption; thus 
proving that $H_{k}$ contains distinct elements. Since $H_{k}$ is contained in $G^{0}$ and $\left|G^{0}\right|=m$ we have $H_{k}=G^{0}$.

Also, since $G^{0}$ is an AG-groupoid under (o) with the left identity $e$, so is $H_{k}$ and hence $H_{k}$ contains the left identity $e$. So, $e$ will be of the form $a_{i} \circ a_{j}$, that is, $e=a_{i} \circ a_{j}$ implying that $a_{i}$ is the left inverse of $a_{j}$ under the binary operation (०). But in an AG-groupoid with left identity, if it contains left inverses, every left inverse is a right inverse. Thus $a_{j}$ is the right inverse of $a_{j}$ under (०).

Since $k=1,2, \ldots, m$ has been chosen arbitrarily, we have shown that $G^{0}$ is an AGgroupoid with left identity and inverses under the binary operation (०).

If $a_{i}, a_{j}, a_{k} \in G^{0}$ such that $a_{i} \circ a_{k}=a_{j} \circ a_{k}$, then $\left(a_{i} \circ a_{k}\right) \circ a_{k}^{-1}=\left(a_{j} \circ a_{k}\right) \circ a_{k}^{-1}$ implies that $\left(a_{k}^{-1} \circ a_{k}\right) \circ a_{i}=\left(a_{k}^{-1} \circ a_{k}\right) \circ a_{j}$ and so $a_{i}=a_{j}$. Thus $G^{0}$ is right cancellative under (०). But $G^{0}$ being right cancellative under $(\circ)$, is left cancellative also, therefore $G^{0}$ is cancellative. Since $G^{0}$ is cancellative whose elements satisfy condition (v), therefore by applying Theorem 2.1, we conclude that $G^{0}$ is a commutative group under $(\circ)$.

COROLlary 2.3. If $(G, \circ)$ is a finite AG-groupoid with left identity and a left zero $a_{0}$, then $\left(G \backslash\left\{a_{0}\right\}, \circ\right)$ is a cancellative AG-groupoid with left identity and inverses provided there is another binary operation $(*)$ such that

(i) $(G, *)$ is an AG-groupoid with left identity and left inverses,

(ii) $a_{0} * a=a$, for all $a \in G$,

(iii) $(a * b) \circ c=(a \circ c) *(b \circ c)$, for all $a, b, c \in G$,

(iv) $a \circ b=a_{0}$ implies that either $a=a_{0}$ or $b=a_{0}$ for all $a, b \in G$.

Proof. The proof is analogous to the proof of Theorem 2.2.

AcKnowledgement. The authors are grateful to the referee for his invaluable suggestions.

\section{REFERENCES}

[1] P. Holgate, Groupoids satisfying a simple invertive law, Math. Student 61 (1992), no. 1-4, 101-106. MR 95d:20113. Zbl 900.20160.

[2] M. A. Kazim and M. Naseeruddin, On almost semigroups, Aligarh Bull. Math. 2 (1972), 1-7. MR 54\#7662. Zbl 344.20049.

[3] Q. Mushtaq and Q. Iqbal, Decomposition of a locally associative LA-semigroup, Semigroup Forum 41 (1990), no. 2, 155-164. MR 91f:20067. Zbl 682.20049.

[4] Q. Mushtaq and M. S. Kamran, On LA-semigroups with weak associative law, Sci. Khyber 2 (1989), no. 1, 69-71.

[5] Q. Mushtaq and S. M. Yusuf, On LA-semigroups, Aligarh Bull. Math. 8 (1978), 65-70. MR 84c:20086. Zbl 509.20055.

[6] P. V. Protić and M. Božinović, Some congruences on an AG**-groupoid, Filomat (1995), no. 9, part 3, 879-886. MR 97b:20097. Zbl 845.20052.

QAISER MuShTAQ: DePARTMENT OF MATHEMATICS, QUAID-I-AZAM UNIVERSITY, ISLAMABAD, PAKISTAN

E-mail address: qmushtaq@apo110.net.pk

M. S. KAMran: Department of MATHEMATICS, QuAid-I-AZAm University, Islamabad, PAKISTAN 


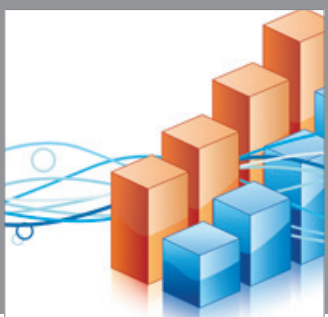

Advances in

Operations Research

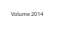

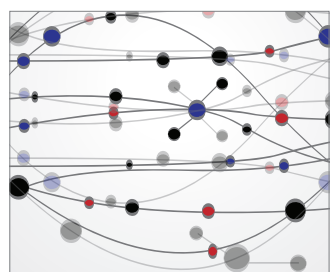

\section{The Scientific} World Journal
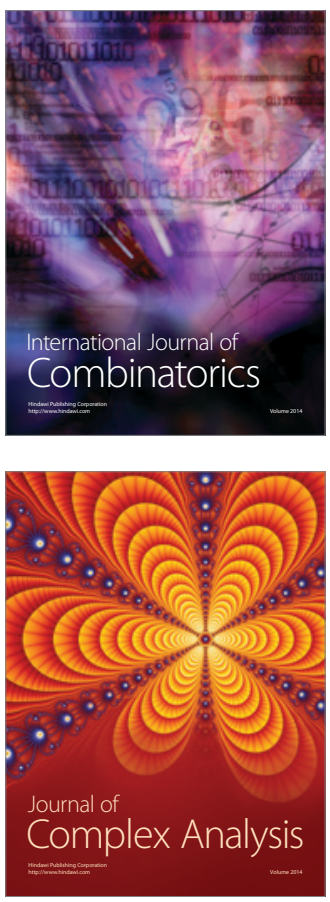

International Journal of

Mathematics and

Mathematical

Sciences
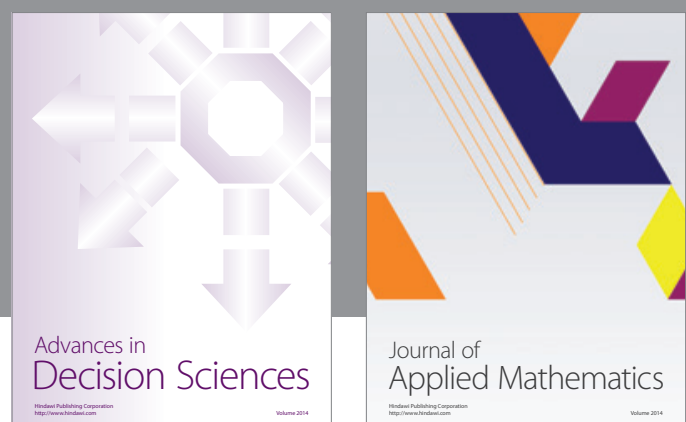

Journal of

Applied Mathematics
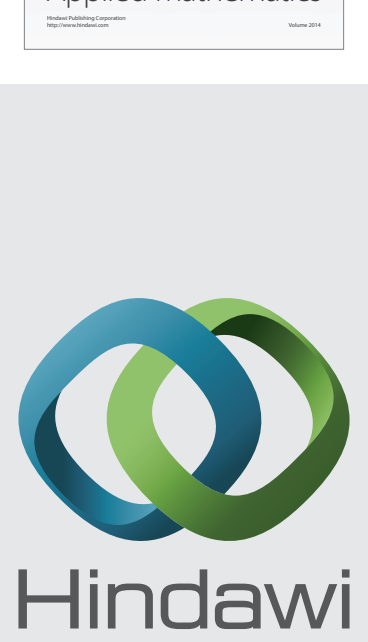

Submit your manuscripts at http://www.hindawi.com
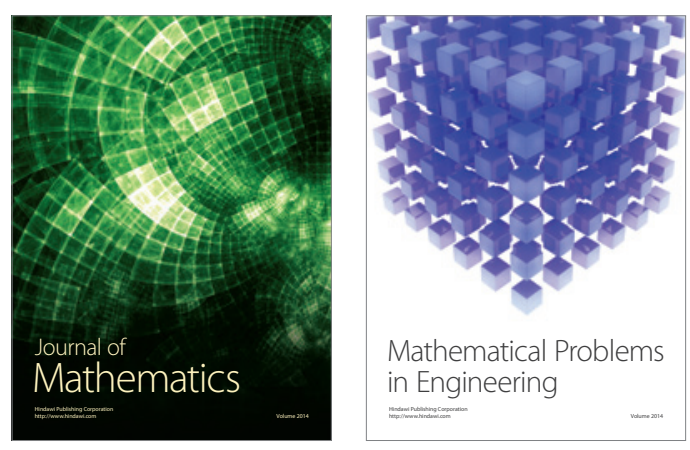

Mathematical Problems in Engineering
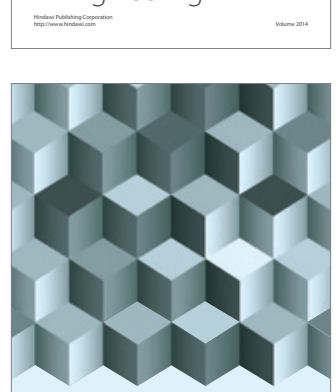

Journal of

Function Spaces
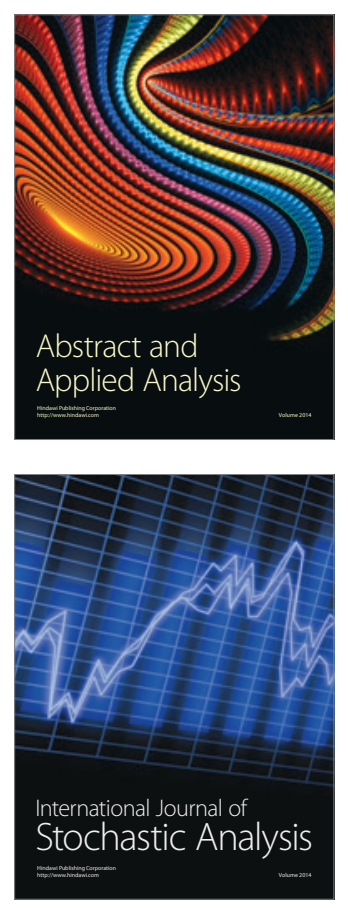

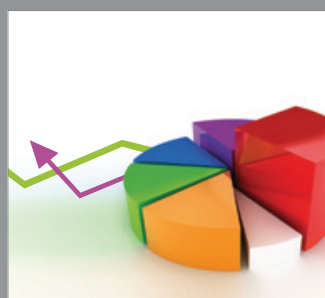

ournal of

Probability and Statistics

Promensencen
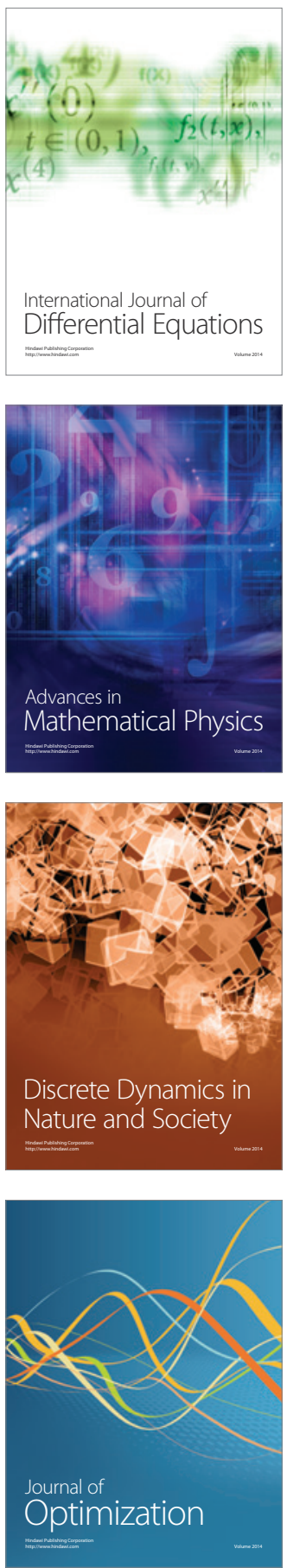